\title{
High Van Hove singularity extension and Fermi velocity increase in epitaxial graphene functionalized by gold clusters intercalation
}

\author{
M. N. Nair ${ }^{1}$, M. Cranney ${ }^{1}$, F. Vonau $^{1}$, D. Aubel ${ }^{1}$, P. Le Fèvre ${ }^{2}$, \\ A. Tejeda ${ }^{2,3}$, F. Bertran ${ }^{2}$, A. Taleb-Ibrahimi ${ }^{2}$ and L. Simon ${ }^{1 *}$ \\ ${ }^{1}$ Institut de Sciences des Matériaux de Mulhouse IS2M-LRC 7228-CNRS-UHA 4, rue des frères Lumière 68093 Mulhouse-France \\ ${ }^{2}$ Synchrotron SOLEIL, L'Orme des Merisiers, Saint-Aubin, 91192 Gif sur Yvette, France and \\ ${ }^{3}$ Institut Jean Lamour, CNRS-Université de Nancy-UPV-Metz, 54506 Vandoeuvre les Nancy, France
}

(Dated: September 25, 2018)

\begin{abstract}
Gold intercalation between the buffer layer and a graphene monolayer of epitaxial graphene on $\mathrm{SiC}(0001)$ leads to the formation of quasi free standing small aggregates of clusters. Angle Resolved Photoemission Spectroscopy measurements reveal that these clusters preserve the linear dispersion of the graphene quasiparticles and surprisingly increase their Fermi velocity. They also strongly modify the band structure of graphene around the Van Hove singularities (VHs) by a strong extension without charge transfer. This result gives a new insight on the role of the intercalant in the renormalization of the bare electronic band structure of graphene usually observed in Graphite and Graphene Intercalation Compounds.
\end{abstract}

PACS numbers: 68.65.-k, 81.16.Fg, 81.07.-b, 81.16.Rf, 82.30.RS, 82.65.+r

The enormous craze for graphene is due to the coexistence between the fundamental aspects of research and the increasing number of potential applications. From the fundamental point of view, this system brings together the physics of particles with relativistic behaviors and the condensed matter. It becomes a promising material for the next generation of nanoelectronic devices destined to supplant silicon [1]. However the main drawback limiting the potential use of graphene stems from its intrinsic characteristics: a semiconductor with zero gap, almost inert towards controlled chemisorption and doping. One of the main challenges is to functionalize the graphene layer while preserving its fascinating properties. Different ways of functionalization have been opened. Deposition of metal or molecules on top of graphene could allow to modify the Fermi level or to induce long range superconductive correlations (for example using superconducting metal contact) [2, 3]. It is also possible to intercalate metal clusters or molecules between the graphene layers, opening the possibility to functionalize the graphene layer on both sides creating a Graphene-Based Hybrid structure (GBHs). In the latter case, the potentialities of modification of the graphene band structure by intercalation meet the historical research community of the Graphite Intercalation Compounds (GICs), well-known in the community of Carbon and also for its famous application which is the Li-ion battery. The research in this field has been considerably intensified after the recent discovery

*corresponding author

Email address: laurent.simon@uha.fr of high Tc superconductivity for the GIC $\mathrm{CaC}_{6}$ [4]. Despite this intense activity, it is still not yet clear if the superconductivity is due to the nature of the intercalant or to the graphene plane itself. Angle Resolved Photoemission Spectroscopy (ARPES) measurements have revealed a systematic VHs extension for these graphitic superconductor [5]. Moreover, recently, in the case of $\mathrm{CaC}_{6}$ the superconducting property has been associated to Charge Density Waves (CDW) evidenced by STM [6]. In this context, epitaxial graphene consists of a playground to understand how the band structure of graphene could be modified and more particularly the various many-body phases that we could expect near VHs. Indeed, the growth of graphene monolayer on silicon face of silicon carbide substrate leads to the formation of a monolayer graphene covalently bonded to the substrate (called buffer layer) which decouples the true monolayer graphene in weak interaction with it. An intercalation process is then possible between these two layers. As the transition from the monolayer to bilayer and few layers graphene can be done in a controlled way on $\mathrm{SiC}(0001)$, this system is also particularly interesting to follow the staging sequences of the intercalation process. In a detailed study by Scanning Tunneling Microscopy (STM), we have revealed that upon specific preparation procedure gold intercalates in two different structures [7]. One is the formation of small intercalated clusters. We have shown by STM that these intercalated gold clusters create a strong "standing waves-like" pattern, on the upper monolayer graphene which has been attributed to a possible VHs singularity extension [8].

The goal of the present work was to realize homogeneous

Typeset by REVTEX 
surfaces with intercalated gold clusters and to explore the band structure of this functionalized graphene on the occupied states with ARPES measurements. We report here ARPES and STM studies of pristine epitaxial graphene and with the intercalation of gold clusters.

The graphene samples were prepared in UHV by the annealing of $\mathrm{n}$-doped $\mathrm{SiC}(0001)$ at $900 \mathrm{~K}$ for several hours and subsequent annealing at $1500 \mathrm{~K}$ [9-11]. The deposition of gold on graphene was carried out at room temperature using a homemade Knudsen cell calibrated using a Quartz Crystal Microbalance. The sample was further annealed at $1000 \mathrm{~K}$ for $5 \mathrm{~min}$ [7]. In order to avoid frequent confusion, we would like to notice that the literature reports two types of intercalation process. One occurs during the annealing process of the carbon rich $\mathrm{SiC}(0001)$ reconstruction in presence of a foreign element for example $\mathrm{H}, \mathrm{F}$ or $\mathrm{Au}[12-16]$. This leads to the intercalation between the $\mathrm{SiC}$ substrate and the $\mathrm{C}$-rich initially covalently bonded graphene layer leading to a partial decoupling. This is associated to a p-type doping effect. In our case the deposition of gold is done after the complete realization of the graphene monolayer leading to the intercalation between the top graphene layer and the buffer layer. Our STM experiments were performed with a LT-STM from Omicron at $77 \mathrm{~K}$ at a base pressure in the $10^{-11}$ mbar range. The $d I / d V$ images were acquired using a lock-in amplifier and a modulation voltage of $\pm 20 \mathrm{mV}$. The ARPES measurements were carried out on the CASSIOPEE beamline of the SOLEIL synchrotron radiation source using a Scienta R4000 electron spectrometer. The spectra were recorded at a photon energy of $60 \mathrm{eV}$ with an overall energy resolution of around $30 \mathrm{meV}$ at a temperature of $10 \mathrm{~K}$. All the samples were prepared in a separated UHV system and then characterized by STM. They were then transported in air prior to their introduction in the UHV system of the synchrotron radiation source. A soft degassing process at $500 \mathrm{~K}$ was performed during several minutes prior to measurements.

Figure 1 shows the resulting STM images of the epitaxial graphene monolayer with the deposition of gold atoms followed by annealing process as previously described. ARPES measurements require surfaces as homogeneous as possible, which has been done here. We are able to control the deposition and annealing process in order to obtain a fairly homogeneous surface with the given intercalation process. Indeed figures 1 a) and b) show respectively a large scale STM image of the studied sample and the corresponding scheme of the repartition of the different domains obtained. More than $80 \%$ of the surface is covered with the intercalated gold clusters (IC) . A small part is occupied by pristine monolayer (ML) and by another domain which corresponds to the insertion of a continuous monolayer of gold (AuF). The continuous monolayer of gold has been evidenced by
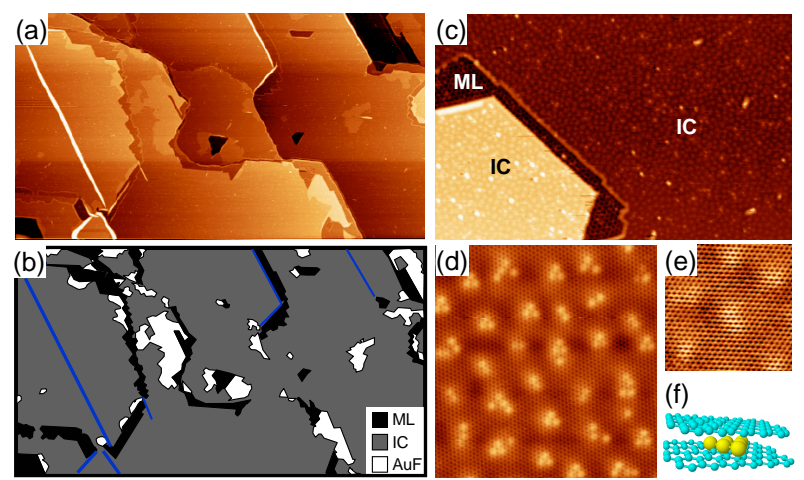

FIG. 1: STM pictures at $77 \mathrm{~K}$ of the surface of epitaxial graphene obtained after $18 \mathrm{ML}$ gold deposition followed by 5 min of annealing at $1000 \mathrm{~K}$. In (a) a large-area topographic image $\left(500 \times 276 \mathrm{~nm}^{2},-1.5 \mathrm{~V}\right)$ and the corresponding scheme (b) below show the repartition of the different domains, where the pristine monolayer graphene domain (ML) is in black, intercalated clusters domain (IC) in grey and gold film domain $(\mathrm{AuF})$ in white (the puckers are in blue). (c) shows the pristine monolayer graphene (ML) and the intercalated clusters region (IC) on two different terraces. The IC region consists of the intercalation of aggregates of flat clusters between the first monolayer graphene and the buffer layer as zoomed in (d) and as schematized in (f). (e) The STM image $\left(5.6 \times 5.6 \mathrm{~nm}^{2}\right.$, $-100 \mathrm{mV}$ ) ascertains that $\mathrm{Au}$ clusters are intercalated below a monolayer graphene; (c) $\left(111 \times 70 \mathrm{~nm}^{2},-1.5 \mathrm{~V}\right)$ and $(\mathrm{d})$ $\left(111 \times 70 \mathrm{~nm}^{2},-1.5 \mathrm{~V}\right)$. (Image processing using the WSxM software [23]).

a Moiré pattern and is associated to a p-doping effect 7]. The bright lines in a) correspond to the initial puckers currently observed on the pristine ML graphene, that are known to be due to the cooling process after the annealing. These defects play probably a role in the intercalation of metal as they are systematically observed on the border of areas with intercalated gold. As shown in figures c) and d), the gold clusters are evidenced by bright protrusion visible at high negative bias (probing the full states). The intercalated gold atoms on the IC domains form a quasi periodically arrangement of aggregates of clusters intercalated between the buffer layer and the top graphene monolayer. Indeed in e) a high resolution image performed at low bias $(-100 \mathrm{meV})$ shows the graphene plane over gold clusters where the 6 carbon atoms of the honeycomb structure are equally visible. This definitively proves that gold clusters are just under the top graphene monolayer. These clusters are less visible for the low bias voltage and the honeycomb structure of graphene dominates the contrast. In Figure d), we tentatively attributed these bright spots to aggregates of flat clusters made of 6 atoms as schematized in figure f) [7]. This proposed model is however still under debate and currently tested by DFT calculations, but the discussion of the exact nature of these clusters is out of the scope of this article. 
We have shown that these clusters create standing waves patterns for bias voltage corresponding to the unoccupied states starting from +0.6 to $+1 \mathrm{eV}[8]$. The non-dispersive character of these standing waves let us consider them as charge density waves (CDW). We have attributed these structures as a screening effect. Indeed we know that in the epitaxial graphene the top Graphene ML is n-doped due to the transfer of charges from the substrate. The Au clusters seem to screen these charges which create a charge inhomogeneity on the graphene plane and scatter the QPs. We have used the Fourier Transform Scanning Tunnelling Spectroscopy Technique (FT-STS) [17] i. e. we performed a 2D FT of the $\mathrm{dI} / \mathrm{dV}$ map images with standing waves pattern in function of the bias voltage. This technique was already successfully used in order to determine the full band structure dispersion of the 2D ErSi $i_{2}$ system [18, 19]. Here the FT-STS showed elliptic features around $\mathrm{M}$ points which have been attributed to a fingerprint of large extension of the VHs. As these results were obtained on the empty states in the band structure, we wanted to explore the filled states with ARPES measurements. In the case of the epitaxied graphene ML on $\mathrm{SiC}(0001)$, the graphene dispersion band is not contaminated by the bulk band structure for a large scale of energy and up to the expected VHs [20].

The experimental spectral functions of our epitaxial ML graphene samples, without and with intercalated gold clusters are shown respectively in figures 2b) and c). Both samples exhibit the characteristic linear dispersion around the K point. The Dirac point is at $230 \mathrm{meV}$ below the Fermi level for the functionalized graphene with gold clusters and $260 \mathrm{meV}$ for the pristine graphene. The doping due to the clusters is quite negligible as previously deduced from STS measurements [7]. For the pristine ML graphene (Fig. $2 \mathrm{~b}$ ), we obtain a very good dispersion characterized by thin bands and linearity over nearly $2 \mathrm{eV}$. The dispersion band indicates that the pristine ML graphene is of very high quality. In the case of graphene with intercalated gold clusters (Fig 2 2 ) the bands are much broader. This is probably due to the reduced size of the homogeneous domains. However the dispersion is also linear until $-2 \mathrm{eV}$. The band exhibits a strong "kink" when approaching the VHs below $-2 \mathrm{eV}$. This is associated to a high increase in the spectral intensity. This is the characteristic of a strong renormalization of the band structure which is usually due to a doping dependence with electron-electron correlation, electronphonon coupling or electron-plasmon coupling. The surprise here comes from the Fermi velocity of the quasiparticles. For the pristine graphene we deduced from the slope a group velocity of $1.02 \pm 0.08 \times 10^{6} \mathrm{~m} . \mathrm{s}^{-1}$ while $1.2 \pm 0.2 \times 10^{6} \mathrm{~m} . \mathrm{s}^{-1}$ is measured in the case of the functionalized graphene with gold. Although the uncertainty
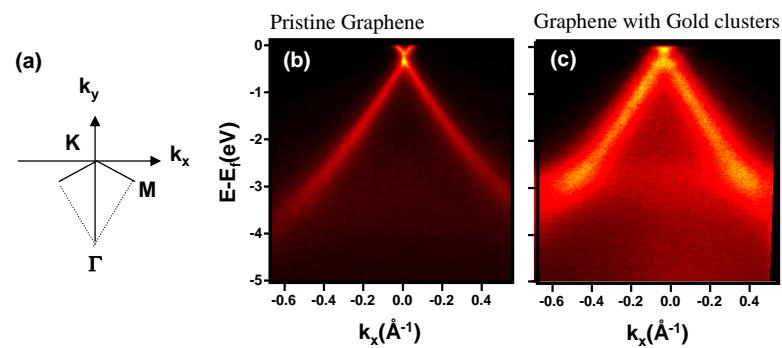

FIG. 2: ARPES intensity of the graphene $\pi$ band around K points along the direction schematized in a). In b) the ARPES measurement is done on the pristine $\mathrm{ML}$ graphene and in c) on the graphene ML with intercalated gold clusters.

is larger in the latter case, this Fermi velocity is increased by more than $20 \%$ of the initial value.

The constant energy surfaces around the $M$ points give also remarkable results. The figure 3 a) recalls the band structure and the interesting topological points of graphene with the calculated Constant Energy Contours (CEC). In b), c) and d) we present respectively the constant energy maps around the M point (which is the position of the expected VHs) at different energies. The VHs are found at $-2.8 \mathrm{eV}$ in the case of pristine graphene in figure $3 \mathrm{c}$ ) and at $-2.3 \mathrm{eV}$ in the case of graphene with gold in fig. [3]). As shown in 3b) the VHs is not reached at $-2.3 \mathrm{eV}$ without the presence of gold clusters. The isocontour in d) (with gold) is strongly warped and the apex of the triangular shaped contour is filled approaching the VHs. These results confirm our interpretation of standing waves pattern and features observed in FT-STS attributed to a large VHs extension [8]. The reason of such a VHs extension remains an open question for the moment. It seems that there is a general behavior of the graphene band structure in the case of intercalated compounds (graphite and epitaxial graphene) particularly around the VHs. Indeed, similar results have been obtained on epitaxial graphene where strong VHs extension has been observed after the intercalation of $\mathrm{K}$ and $\mathrm{Ca}$ in epitaxial graphene layer [21]. In this latest experiment the graphene was strongly n-doped and the Fermi level was positioned at the VHs of the graphene-derived $\pi^{*}$ states which are usually suspected to be at the origin of the superconductivity. Up to now, whatever the studied system (GICs or intercalated graphene), the intercalant was considered to be homogeneously distributed between graphene planes leading to specific surstructures (usually a p-2x2) depending on the stoichiometry 22$]$. However in each of these cases the VHs extension has been associated to a highly doping process and electron-electron correlation. The Fermi velocities were found generally lower $\left(0.5\right.$ to $\left.0.7 \times 10^{6} \mathrm{~m} . \mathrm{s}^{-1}\right)$. In our case the electronelectron correlation has to be ruled out. The sample is no more doped compared to the pristine graphene and the Fermi velocity is increased by the intercalated clus- 
ters. The Fermi velocities, we have measured for Pristine graphene and with intercalated gold clusters are comparable to those found in the literature, i. e. 0.9 to $1 \times 10^{6} \mathrm{~m} \cdot \mathrm{s}^{-1}$ for epitaxial graphene on $\mathrm{SiC}$ for the $\mathrm{Si}$ terminated face and $1.1 \times 10^{6} \mathrm{~m} . \mathrm{s}^{-1}$ for the carbon terminated face (see for example 24]), or in the case of exfoliated graphene on $\mathrm{SiO}_{2}$ 1]. Then the Fermi velocity we have measured with the intercalated gold clusters is comparable to the one measured on graphene epitaxied on the $\mathrm{C}$-face. This tends to demonstrate that the clusters decouple the graphene layer from the substrate as in the case of C- face graphene layers. However this leads to a counterintuitive reasoning. Indeed, among the all possible origins of the VHs extension, the pseudo periodic potential created by the clusters is the most probable hypothesis. Following the theoretical calculation of Cheol et al. 25], applying a weak pseudopotential should leads to decrease the group velocity (renormalization). This work also shows that a slight potential oscillation and/or corrugation on the graphene layer tends to strongly modify the VHs. Here the compressive strain of graphene is probably partially released by the decoupling induced by the clusters, leading to an increase of lattice parameter and consequently an increase of the Fermi velocity compared to pristine graphene. Concerning the VHs extension, one more time this appears to be a general characteristic of the graphene electronic properties. Indeed, as soon as the hopping process of the quasiparticule is modified, either theoretically by the modification of the Hopping potential 26] or experimentally by the modification of the rotation angle between two graphene layers [27], the VHs are affected. In a very complete detailed study of the graphitic superconductors, it has been demonstrated that critical temperature Tc depends on the charge transfer between the intercalated atoms and the graphene plane i.e. the doping level. The increase of Tc was correlated to an expected electron-phonon coupling which is associated to the kink in the dispersion band structure 5]. This was also associated to a strong anisotropic Fermi velocity and trigonal warping in the constant energy contour map around the $\mathrm{K}$ point approaching the Fermi level. The reason why these clusters modify the band structure in this way remains an open question but in this context, considering the importance of the controversial possible role of the VHs in the superconductivity property (VHs extension scenario), particularly in the case of GICs, our results open a new way for the understanding of this phenomena. Indeed here the graphene is not strongly doped, and the intercalant is not homogeneously distributed. The last but not the least point of this study is to show one more time the link between the FT-STS and ARPES measurements, even if it is not discussed in detail [8].

This work is supported by the Région Alsace and the CNRS. The Agence Nationale de la Recherche supports this work under the ANR Blanc program, reference ANR-

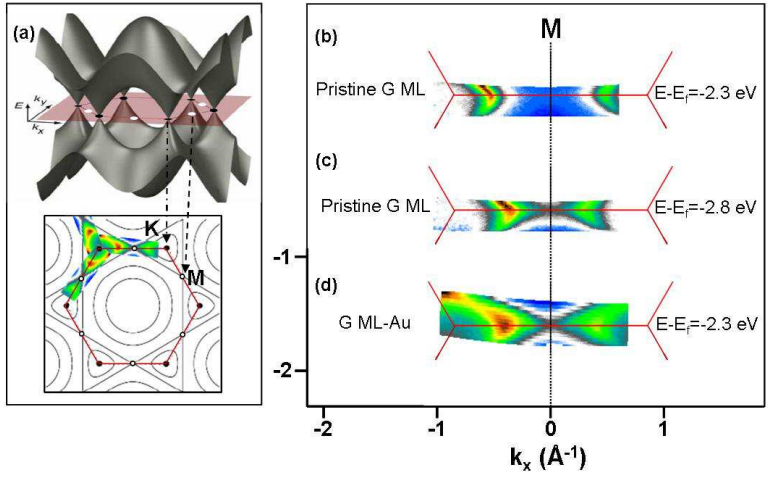

FIG. 3: a) 3D representation of the band structure of graphene and 2D Constant Energy Contours map (CECs). The CECs are calculated in a 1 NN TB approximation. The key features of the band structure i.e. the Dirac points and Van Hove singularities (VHs) are indicated on the 3D representation. b), c) and d, show equienergetic contours of the photoemission intensity around $\mathrm{M}$ points; b) and c)for the pristine graphene at $-2.3 \mathrm{eV}$ and $-2.8 \mathrm{eV}$ respectively, and $\mathrm{d}$ ) for the graphene with intercalated gold clusters at $-2.3 \mathrm{eV}$. The high extension of VHs observed in the case of graphene ML with intercalated gold cluster in d) is clearly seen while for the same energy in b) the VHs is not reached for pristine graphene. The isoenergetical surface obtained by three-fold symmetrization of the contour showed in d) is reported in a).

\section{0-BLAN-1017-ChimiGraphN}

[1] K. S. Novoselov, A. K. Geim, S. V. Morozov, D. Jiang, M. I. Katsnelson, I. V. Grigorieva, S. V. Dubonos, and A. A. Firsov, Nature 438, 197 (2005).

[2] B. M. Kessler, C .Ö. Girit, A. Zettl, and V. Bouchiat, Phys. Rev. Lett. 104, 047001 (2010).

[3] Travis Dirks, Taylor L. Hughes, Siddhartha Lal, Bruno Uchoa, Yung-Fu Chen, Cesar Chialvo, Paul M. Goldbart, and Nadya Mason, Nat. Phys. 7, 386 (2011).

[4] T. Weller, M. Ellerby, S. Saxena, R. Smith, and N. Skipper, Nat. Phys. 1, 39 (2005).

[5] Z.-H. Pan, J. Camacho, M. H. Upton, A. V. Fedorov, C. A. Howard, M. Ellerby, and T. Valla, Phys. Rev. Lett. 106, 187002 (2011).

[6] K.C. Rahnejat, C.A. Howard, N.E. Shuttleworth, S.R. Schofield, K. Iwaya, C.F. Hirjibehedin, Ch. Renner, G. Aeppli, and M. Ellerby, Nat. Commun. 2:558 doi: 10.1038/ncomms1574 (2011).

[7] B.Premlal et al., Appl. Phys. Lett. 94, 263115 (2009).

[8] M. Cranney, F. Vonau, P.B. Pillai, E. Denys, D. Aubel, M.M. De Souza, C. Bena, and L. Simon, EPL 91, 66004 (2010).

[9] A. J. Van Bommel et al, Surf.Sci. 48, 463(1975).

[10] L. Simon, J. L. Bischoff, and L. Kubler, Phys. Rev. B 60, 11653 (1999).

[11] C. Berger, Z. Song, T. Li, X. Li, A. Y. Ogbazghi, R. Feng, Z. Dai, A. N. Marchenkov, E. H. Conrad, P. N. First, and W. A. de Heer, J. Phys. Chem., 108, 19912 
(2004).

[12] A. Bostwick, F. Speck, T. Seyller, K. Horn, M. Polini, R. Asgari,A. H. MacDonald, and E. Rotenberg, Science 328, 999 (2010).

[13] A. L. Walter, K.-J Jeon, A. Bostwick, F. Speck, M. Ostler, T. Seyller, L. Moreschini, Y. S. Kim, Y. J. Chang, K. Horn, and E. Rotenberg, Appl. Phys. Lett. 98, 184102 (2011).

[14] I. Gierz, T. Suzuki, R. T. Weitz, D. S. Lee, B. Krauss, C. Riedl, U. Starke, H. Höchst, J. H. Smet, C. R. Ast, and K. Kern, Phys. Rev. B 81, 235408 (2010).

[15] S. Forti, K. V. Emtsev, C. Coletti, A. A. Zakharov, C. Riedl, and U. Starke, Phys. Rev. B 84, 125449 (2011)

[16] Swee Liang Wong, Han Huang, Yuzhan Wang, Liang Cao, Dongchen Qi, Iman Santoso, Wei Chen and Andrew Thye Shen Wee, ACSNANO 5, 7662 (2011).

[17] L. Simon, F. Vonau and D. Aubel, J. of Phys. Cond. Mat., 19, 355009 (2007).

[18] F. Vonau, D. Aubel, G. Gewinner, S. Zabrocki, J. C. Peruchetti, D. Bolmont, and L. Simon, Phys. Rev. Lett. 95, 176803 (2005).

[19] L. Simon, C. Bena, F. Vonau, M. Cranney and D. Aubel,
J. Phys. D 44, 464010 (2011).

[20] F. Varchon, R. Feng, J. Hass, X. Li, B. Ngoc Nguyen, C. Naud, P. Mallet, J.-Y. Veuillen, C. Berger, E. H. Conrad, and L. Magaud, Phys. Rev. Lett. 99, 126805 (2007).

[21] Jessica L. McChesney, Aaron Bostwick, Taisuke Ohta, Konstantin V. Emtsev, Thomas Seyller, Karsten Horn, and Eli Rotenberg, Phys. Rev. Lett. 104, 136803 (2010).

[22] M.S. Dresselhaus, and G. Dresselhaus Advances in Physics vol 51, p1-186 (2002).

[23] I. Horcas et al, Rev. Sci. Instrum. 78, 013705 (2007).

[24] Th. Seyller, A. Bostwick, K. V. Emtsev, K. Horn, L. Ley, J. L. McChesney, T. Ohta, J. D. Riley, E. Rotenberg, and F. Speck Phys. Stat. Sol. (b) 245, 1436 (2008).

[25] Cheol-Hwan Park, Li Yang, Young-Woo Son, Marvin L. Cohen and Steven G. Louie Nat. Phys. 4, 213 (2008).

[26] Cristina Bena, and Laurent Simon, Phys. Rev. B 83, 115404 (2011).

[27] Guohong Li, A. Luican, J. M. B. Lopes dos Santos, A. H. Castro Neto, A. Reina, J. Kong, and E. Y. Andrei, Nat. Phys. 6, 109 (2010). 